\title{
Isolated tubercular orchi-epididymitis with painful hydrocoele: Case report
}

\author{
NEDJIM Abdelkerim Saleh ${ }^{\mathrm{a}^{*}}$, El IDRISSI Alami Oussama ${ }^{\mathrm{a}}$, GHANNAM Youssef ${ }^{\mathrm{a}}$, ABDI Moustapha ${ }^{\mathrm{a}}$,

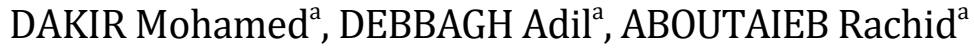

${ }^{a}$ Department of Urology, Ibn Rochd University Hospital and Faculty of Medicine and Pharmacy, Casablanca, Morocco.

\section{Abstract}

Testicular tuberculosis is rare and represents only $3 \%$ of genitourinary tuberculosis cases. We are reporting a case of tuberculous orchi-epididymitis which was manifesting as a painful hydrocoele knowing that the condition has become favourable with a good evolution after conservative surgical intervention and anti-bacillary treatment.

Keywords: Hydrocoele; orchiepididymitis; surgery; tuberculosis

\section{INTRODUCTION}

Testicular tuberculosis is rare about genitourinary tuberculosis and represents only 3\% of genitourinary tuberculosis cases ${ }^{[1]}$. Its clinical symptomatology is non-specific and the available microbiological tests are of low sensitivity. Treatment can be medical at an early stage, but the delay in diagnosis can lead to orchiectomy ${ }^{[2]}$. We are reporting a case of tuberculous orchiepididymitis that has manifested as a painful hydrocoele and the condition has become favorable with a good evolution after conservative surgical intervention and anti-bacillary treatment.

\section{CASE REPORT}

Mr. A. A, 43 years old, a chronic smoker, with no notable past medical history, come to the emergency department for pain with fever on scrotal swelling that had been evolving for about one year. Clinical examination found a conscious and stable patient with a body

* Corresponding author: NEDJIM Abdelkerim Saleh Mailing address: Department of Urology, Ibn Rochd University Hospital and Faculty of Medicine and Pharmacy, Casablanca, Morocco.

E-mail: nedjimsaleh@gmail.com

Received: 11 October 2020 / Accepted: 11 November 2020 temperature of $37.8{ }^{\circ} \mathrm{C}$ and painful right scrotal swelling extending to the inguinal region. This swelling was transluminal (Figure 1).

The ultrasound scan (Figure 2) carried out as a matter of emergency revealed the presence of a scrotal swelling which was poorly limited with thick and mobile echogenic content. This thick formation extends toward the right spermatic cord which is swollen and oedematous. It is associated with a right testicle of normal volume, regular contours, heterogeneous echostructure with a poorly limited hypoechoic range and hypervascularization.

Surgical exploration revealed a hydrocele with a cloudy content and significant swelling of the cord with the presence of fibrous tissue at the expense of the right testicle (Figure 3). The epididymis was individualized. Fluid sampling and biopsies were taken and a hydrocoele cure was performed. The Ziehl stain was positive, and the cytology has isolated tuberculoid granulomas. The postoperative follow-up was simple. Bacteriological and cytological studies supported the diagnosis of tuberculosis. The patient was put under anti-bacillary treatment.

\section{DISCUSSION}

Genitourinary tuberculosis involving the epididymis is caused by retrograde extension from the prostate 
and/or seminal vesicles, or by blood. The epididymal tail is most often affected, and the involvement may be unilateral or bilateral. Untreated epididymal infection can progress to orchi-epididymitis. Tuberculous involvement may be manifested by the thickening of the scrotal skin, hydrocoele, or scrotal abscess ${ }^{[3]}$. Testicular involvement is rare and represents only $3 \%$ of genitourinary tuberculosis cases ${ }^{[1]}$. We are reporting one case of isolated tuberculous orchi-epididymitis with a painful hydrocoele as a presentation.

In $80 \%$ of the cases, patients with tuberculous orchiepididymitis have a scrotal mass, which can be painful in $40-44 \%$. Bilateral tuberculous involvement is observed in $34 \%$ of cases, $4-50 \%$ may present late with an abscess or fistula and $5-10 \%$ may have an associated hydrocoele ${ }^{[4]}$. It has been described that the presentation appears as a painful, rapidly progressing hydrocoele that's due to the isolated involvement of the albuginea and tunica vaginalis ${ }^{[5]}$.

Ultrasonography is currently the best imaging technique to study the scrotum and its contents. It can be used to reliably differentiate between intratesticular lesions ${ }^{[6]}$. Ultrasound can reveal heterogeneous epididymitis with hypoechoic areas ${ }^{[7]}$. The ultrasound carried out in our patient's emergency revealed the presence of a poorly limited scrotal swelling with a thick and mobile echogenic content. In addition to the aspects described in the literature, the right spermatic cord was swollen and oedematous with the contralateral testis which is heterogeneous and has a poorly limited hypoechoic area with hypo-vascularisation.

The diagnosis can be confirmed by culture, ZiehlNeelsen staining, and/or histological examination ${ }^{[8]}$. The cytological study allowed the diagnosis to be made

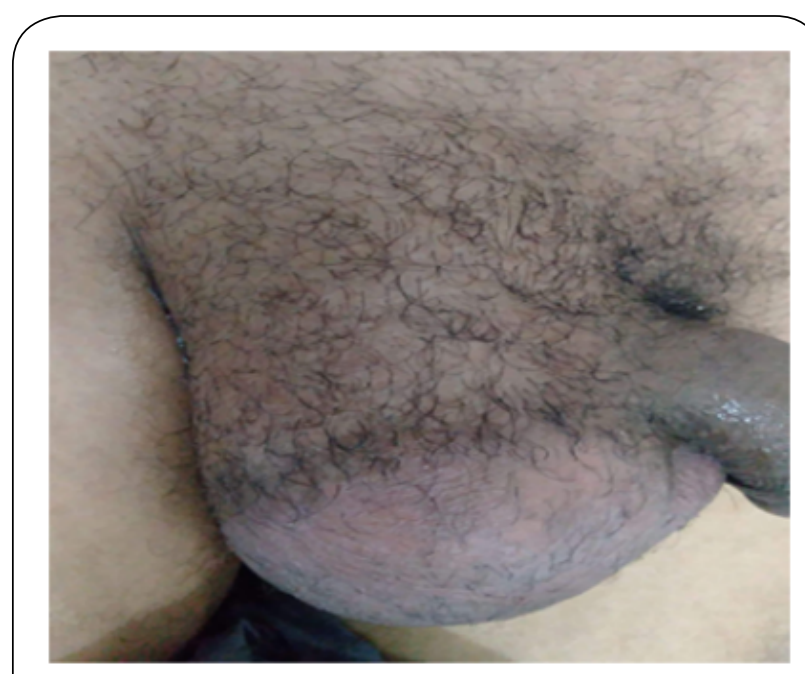

Figure 1. A scrotal swelling which is painful and transluminal.
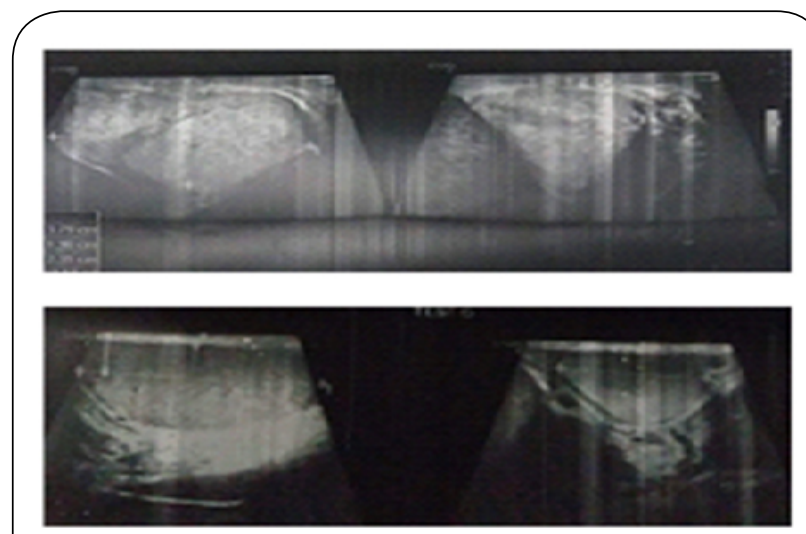

Figure 2. Testis and scrotum swelled with echoes heterogeneous structure and hypoechogenic areas.

by isolating a tuberculoid granuloma with a positive Ziehl stain.

The treatment of tuberculous orchi-epididymitis is es-

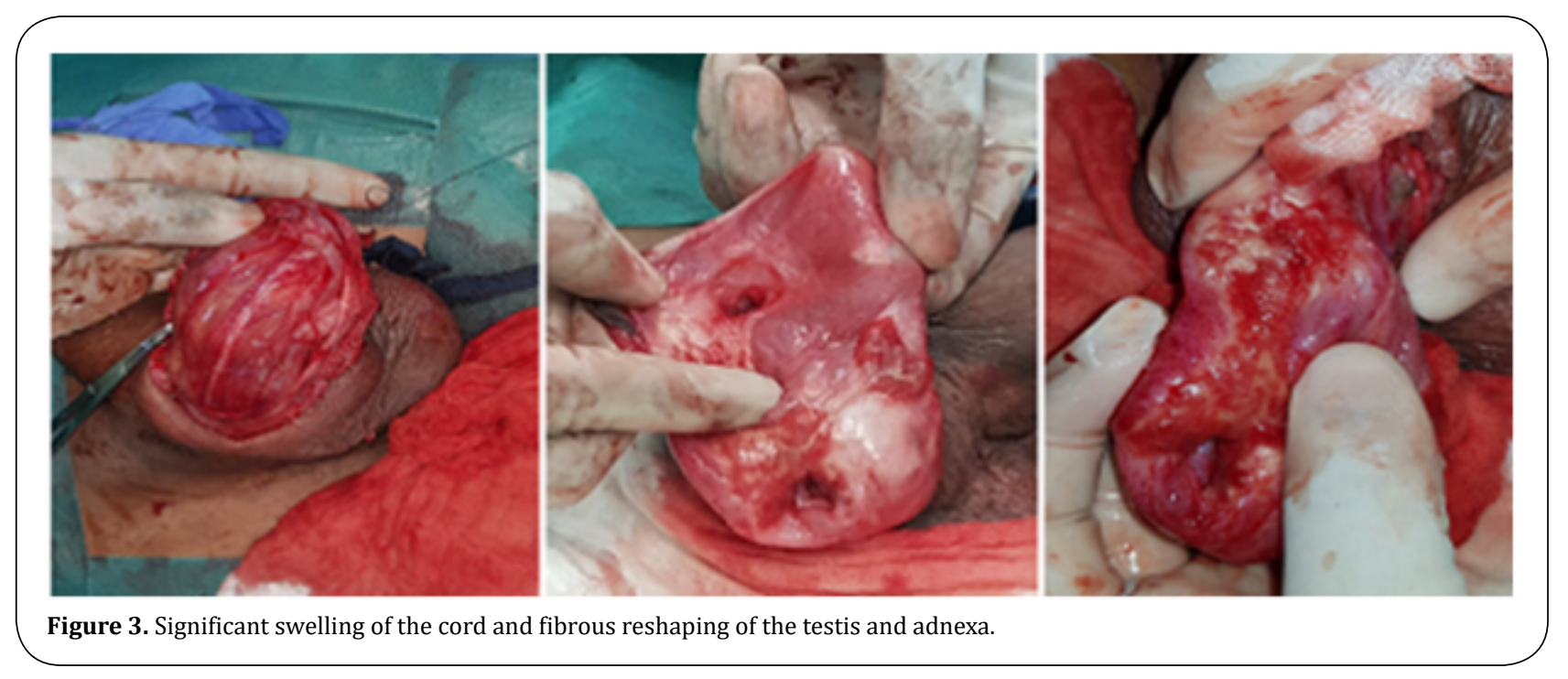


sentially conservative. The classic scheme in practice is the combination of four molecules (rifampicin, isoniazid, pyrazinamide, and ethambutol) for 6 months in two phases. The cure rate is over $95 \%{ }^{[9]}$. Surgery is recommended for patients who do not respond to medical treatment within the first 2 months or those who present an intrascrotal abscess ${ }^{[10]}$. Our attitude was conservative. The evolution was favorable after six months of anti-bacillary treatment.

\section{CONCLUSION}

Genital tuberculosis is rare and not often mentioned in practice. In front of a painful and feverish hydrocoele, the hypothesis deserves to be evoked. The prognosis depends on the diagnosis and treatment.

\section{DECLARATIONS}

\section{Authors' contributions}

NEDJIM and El IDRISSI contributed to all stages of the article.

GHANNAM and ABDI contributed to the bibliographical research .

DAKIR, DEBBAGH and ABOUTAIEB corrected the article.

\section{Conflict of interest}

All the authors stated that there is no conflict of interest.

\section{Ethical approval and consent to participate}

Not applicable.

\section{Consent for publication}

Not applicable.

\section{REFERENCES}

1. Das, A., Batabyal, S., Bhattacharjee, S., \& Sengupta, A. (2016). A rare case of isolated testicular tuberculosis and review of literature. Journal of family medicine and primary care, 5(2), 468.

2. Yadav, S., Singh, P., Hemal, A., \& Kumar, R. (2017). Genital tuberculosis: current status of diagnosis and management. Translational Andrology and Urology, 6(2), 222.

3. Muttarak, M., Peh, W. C., Lojanapiwat, B., \& Chaiwun, B. (2001). Tuberculous epididymitis and epididymoorchitis: sonographic appearances. American Journal of Roentgenology, 176(6), 1459-1466.

4. Suankwan, U., Larbcharoensub, N., Viseshsindh, W., Wiratkapun, C., \& Chalermsanyakorn, P. (2012). A clinicopathologic study of tuberculous epididymo-orchitis in Thailand. Southeast Asian Journal of Tropical Medicine \& Public Health, 43(4), 951-958.

5. Khan, S., Haroon, N., Azami, R., \& Bawa, T. (2015). Isolated tuberculosis of tunica albuginea and tunica vaginalis presenting as acute hydrocoele: a diagnostic dilemma. Case Reports, 2015, bcr2014207744.

6. Dell'Atti, L. (2013). Ultrasound diagnosis of unusual extratesticular mass: case report and review of the literature. Archivio Italiano di Urologia e Andrologia, 85(1), 41-43.

7. Chung, J. J., Kim, M. J., Lee, T., Yoo, H. S., \& Lee, J. T. (1997). Sonographic findings in tuberculous epididymitis and epididymo-orchitis. Journal of clinical ultrasound, 25(7), 390-394.

8. Lenk, S., \& Schroeder, J. (2001). Genitourinary tuberculosis. Current opinion in urology, 11(1), 93-96.

9. Chan, E. D., \& Iseman, M. D. (2002). Current medical treatment for tuberculosis. Bmj, 325(7375), 1282.

10. Gow, J. G., \& Barbosa, S. (1984). Genitourinary tuberculosis. A study of 1117 cases over a period of 34 years. British journal of urology, 56(5), 449-455. 